\title{
ESTIMACIÓN DE LA MÍNIMA PRESIÓN DE MISCIBILIDAD EN LAS ARENAS BASALES DEL CAMPO COLOMBIA EN PAZ
}

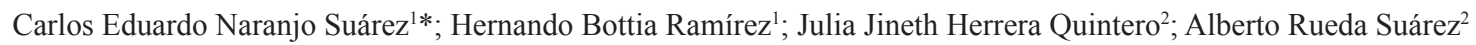 \\ ${ }^{1}$ Empresa Colombiana de Petróleos. Ecopetrol. Vía Piedecuesta Km 7. Piedecuesta, Colombia \\ ${ }^{2}$ Universidad Industrial de Santander (UIS). Carrera 27 Calle 9. Bucaramanga, Colombia. \\ *E-mail: carlosed.naranjo@ecopetrol.com.co
}

\section{RESUMEN}

En el Campo Colombia en paz se visualiza la oportunidad de incrementar productividad mediante la implementación de métodos de recobro secundario. El proyecto apunta a las arenas basales debido a que la calidad de su fluido es mejor en comparación con las arenas superiores ( $30^{\circ} \mathrm{API}$ vs $20^{\circ} \mathrm{API}$, de forma respectiva). El objetivo principal es evaluar el efecto de la reinyección de gas en la producción de petróleo.

En la actualidad el campo dispone de una red de gas para levantamiento artificial (Gas Lift) con una presión disponible de 1,200 psi. Esta red se usaría para la implementación del proceso, tanto de manera cíclica como continua, con tasas de flujo entre 100 y 1,000 kscfpd (kilo standard cubic feet per day). Se espera que la producción de petróleo se incremente debido a la presurización en el área de drenaje del pozo e incremento de la movilidad por solubilidad parcial del gas en el crudo.

En el Centro de Innovación y Tecnología - Instituto Colombiano del Petróleo se midió el recobro de crudo por la inyección de gas de producción. Para ello, se siguió el instructivo técnico de ensayo GTN-I-009 para realizar evaluaciones con gas de producción. Los ensayos se realizaron a temperatura de yacimiento de $138^{\circ} \mathrm{F}$, Gas Oil Ratio (GOR) de 440 scf/STB y crudo con agua y sedimentos Basic Sediments and Water (BS\&W) menor que $1 \%$, tal como lo exigen los protocolos experimentales.

En laboratorio, la eficiencia de desplazamiento de crudo con gas de producción fue del $18 \%$ a la presión máxima de operación del sistema Gas Lift de 1,600 psi. La Mínima Presión de Miscibilidad se estimó en 8,407 psi mediante extrapolación lineal debido a que se tomaron datos a dos presiones en el equipo Slim Tube.

Palabras claves: Inyección de gas, Slim Tube, Mínima Presión de Miscibilidad.

\section{MINIMUM MISCIBILITY PRESSURE ESTIMATION IN THE LOWER SANDS OF COLOMBIA EN PAZ OILFIELD}

\begin{abstract}
In Colombia en paz Oil Field, the opportunity to increase productivity through the implementation of secondary recovery methods is visualized. The project aims at basal sands because the quality of its fluid is better compared to the upper sands ( $30^{\circ}$ API vs $20^{\circ} \mathrm{API}$, respectively). The main objective is to evaluate the effect of gas reinjection on oil production.

Today, the field has a gas lift network (Gas Lift) with an available pressure of 1,200 psi. Which would be used for the implementation of the process both cyclic and continuous and with flow rates between $100-1,000 \mathrm{kscfpd}$ (kilo standard cubic feet per day). Oil production is expected to increase due to pressurization in the well drainage area and increased mobility by partial solubility of the gas in the crude.

At the Center of Innovation and Technology - Instituto Colombiano del Petróleo, the recovery of crude oil was measured by the injection of production gas. The technical standard GTN-I-009 was used to conduct runs with production gas. The tests were performed at $138^{\circ} \mathrm{F}$ reservoir temperature, $440 \mathrm{scf} / \mathrm{STB}$ Gas Oil Ratio and crude with Basic Sediments and Water (BS\&W) less than $1 \%$ as required by the experimental protocols.
\end{abstract}

Cita: Naranjo, C., Bottia, H., Herrera, J. y Rueda, A. (2017). Estimación de la mínima presión de miscibilidad en las arenas basales del campo Colombia en Paz. Revista Fuentes: El reventón energético, 15 (1), 101-107. 
At the laboratory, the total recovered crude with production gas was $18 \%$ at the maximum operating pressure of 1,600 psi for the Gas Lift system. The Minimum Miscibility Pressure was estimated at 8,407 psi by linear extrapolation using experimental data taken from Slim Tube Equipment .

Key words: Gas injection, Slim Tube, Minimum Miscibility Pressure.

\section{INTRODUCCIÓN}

En el Centro de Innovación y Tecnología, Instituto Colombiano del Petróleo, se cuantificó el recobro de crudo del Campo Colombia en paz con gas de producción. Estas pruebas de laboratorio hacen parte de un estudio de factibilidad para implementar un proceso de recobro secundario por reinyección de gas en las arenas basales de la Formación productora. La primera etapa de dicha evaluación comprendió la toma de muestras de petróleo y gas y su respectiva caracterización fisicoquímica y análisis de Presión, Volumen y Temperatura (PVT).

En una segunda etapa se realizaron las evaluaciones experimentales para determinar el recobro total de crudo. En la actualidad la presión promedia de yacimiento está en el rango de 700-800 psi, pero el valor disponible en la red del sistema Gas Lift es mayor y cercana a 1,200 psi. Lo cual favorecería el proyecto debido a que no se requerirían inversiones adicionales en compresión para la implementación del proceso.

Otro punto a favor del proyecto, además de la infraestructura ya existente en campo, tiene que ver con la calidad y disponibilidad de fluidos. El yacimiento candidato, arenas basales, produce un crudo con alta gravedad API, alrededor de 30 grados, lo cual haría factible el proceso de incremento en recobro. El activo también reporta un excedente de gas de producción, el cual tiene un contenido de metano alrededor del $90 \%$.

\section{PlanteAMIENTO DEL PROBLEMA E HIPÓTESIS}

En las condiciones actuales del campo no es posible pensar en la implementación de un proceso miscible. La presión de yacimiento está alrededor de los 700800 psi y este valor se considera bajo si se compara con su presión de burbuja, la cual oscila entre 1,700 y 1,850 psi. Otro factor en contra es que la red Gas Lift se diseñó para operar a una presión máxima de 1,600 psi. Es decir, tampoco se cuenta con la energía de compresión necesaria para apuntarle a un proyecto de esta naturaleza.

Sin embargo, se espera que la reinyección del gas genere un incremento en la productividad debido a dos mecanismos principales. El primero, es la represurización en el área de drenaje de los pozos estimulados por la inyección cíclica (Huff and Puff). El segundo, es una mejora de la movilidad por efecto de la solubilidad parcial del gas reinyectado en el petróleo. Esto se basa en el hecho de que la presión en el área de drenaje se puede incrementar hasta 1,600 psi, valor cercano a la presión de burbuja.

\section{OBJETIVOS}

\section{OBJETIVO GENERAL}

Cuantificar la eficiencia de desplazamiento de crudo por la inyección de gas de producción en Equipo Slim Tube.

\section{OBJETIVOS ESPECÍFICOS}

1. Determinar la eficiencia de desplazamiento de crudo con gas de producción a la presión máxima de operación del sistema Gas Lift.

2. Estimar la Mínima Presión de Miscibilidad del gas de producción en el crudo del mismo campo.

\section{MÍNIMA PRESIÓN DE MISCIBILIDAD-MPM}

La inyección de gases es el proceso EOR (Enhanced Oil Recovery) de mayor aplicación mundial en crudos livianos y medios (Zhang, 2016) (Zhang, et al., 2016) (Al-Khazraji \& Awang, 2016). Los más utilizados son los hidrocarburos producidos del mismo yacimiento o fluidos exógenos como el dióxido de carbono, aunque también se reportan algunos proyectos con nitrógeno (2016 Enhanced Oil Recovery (EOR) Dataset) (BP Statistical Review of World Energy, 2016).

Dichos procesos de recobro pueden ser aplicaciones miscibles o inmiscibles. En el primer caso, se presenta vaporización de los componentes más livianos del crudo en el gas de inyección y lo hacen más rico. También, de manera simultánea, se presenta la condensación de las fracciones más pesadas del gas en el líquido y se forma una zona de transición la cual se hace cada vez más miscible tanto con la fase gaseosa enriquecida como con el crudo medios (Zhang, 2016).

Este proceso se repite una y otra vez a través del tiempo hasta que se alcanza una mezcla homogénea 
y estable. Razón por la cual se denomina miscibilidad por múltiples contactos (Multiple-Contact MiscibilityMCM). Pero, cuando los crudos contienen una alta relación de compuestos livianos se podría afirmar que el gas de producción sería miscible al primer contacto (First Contact Miscibility - FCM) y no se presentaría el fenómeno de la formación de una zona de transición en la interfaz líquido gas.

Una vez se logra miscibilidad se observa el fenómeno de hinchamiento del petróleo. Cambia su composición química y se tiene un fluido más rico y de mayor valor. También se reduce su viscosidad, lo cual hace que mejore su movilidad, aumentando así el barrido volumétrico. Otro efecto muy importante es que se reduce la tensión interfacial, lo cual a su vez conduce a que se reduzca la saturación residual de crudo medios (Zhang, 2016) (Zhang, et al., 2016) (Cho, Kim \& Lee, 2016) (Rommerskirchen, Nijssen, Bilgili \& Sottmann, 2016) (Ennin \& Grigg, 2016) (Al-Khazraji \& Awang, 2016).

En un proceso inmiscible los fluidos no se mezclan de ninguna forma entre ellos y no se forma un nuevo fluido. En ese caso los principales mecanismos son incremento en presión y reemplazo físico del petróleo por el gas inyectado (Rommerskirchen, et al., 2016) (Adel, Tovar \& Schechter, 2016). Sin embargo, el recobro siempre será mayor cuando el proceso se implementa de manera miscible (Zhang, 2016) (Zhang, et al., 2016) (Cho, et al., 2016).

Para la determinación de la mínima presión de miscibilidad, la literatura reporta cinco (5) metodologías. Ellas son: celda de burbuja ascendente (Rising Bubble Apparatus - RBA), pruebas de tubo delgado (Slim Tube Testing), análisis de tension interfacial (Interfacial Tension - IFT analysis), pruebas en columna empacada (Packed Column Testing) y pruebas de desplazamiento en medio poroso (Linear Core Flooding) (Trost P. B., 2016).

Sin embargo, el equipo más usado a nivel mundial es el Slim Tube. Consta de un tubo de acero inoxidable enrollado en forma de espiral con una longitud total de 60 pies. La cual es suficiente para que se mezclen en su totalidad los fluidos sin importar si el proceso es miscible al primer o por múltiples contactos. Al mismo tiempo la velocidad de desplazamiento debe ser baja para que se presente dispersión axial y radial y se evite la canalización del gas (Adel, et al., 2016). Se considera que se ha alcanzado la mínima presión de miscibilidad cuando el recobro es igual o superior al $95 \%$ después de la inyección de 1.25 volúmenes porosos de gas (Rommerskirchen, et al., 2016).
El equipo de miscibilidad Slim Tube es un medio poroso ideal en el cual se evalúa el desempeño termodinámico del proceso. Allí no se tienen en cuenta varios fenómenos que se podrían presentar durante la implementación de un proyecto de esta naturaleza en yacimiento, tales como digitación, canalización, dispersión del frente de desplazamiento, segregación gravitacional, entre otros (Al Saadi H., et al., 2016; Cho, et al., 2016).

Por lo anterior, los resultados experimentales no se deben interpretar como factores de recobro escalables a campo. Ellos son sólo una valoración cualitativa del efecto de la implementación de este tipo de procesos en la respuesta en producción del yacimiento. Es por ello que un proyecto de esta naturaleza debe incluir otros análisis adicionales tales como desplazamientos en núcleos nativos, estudios de comportamiento de fases, simulación composicional, numérica o analítica, análisis de costos, entre otros.

\section{METODOLOGÍA EXPERIMENTAL}

Las evaluaciones se realizaron con base en lineamientos establecidos en instructivos del Centro Innovación y Tecnología Instituto Colombiano del Petróleo. El documento con identificación interna GTN-I-009 contiene el paso a paso para determinar la mínima presión de miscibilidad de un gas en un crudo. El procedimiento GTN-I-019 establece las actividades a ejecutar en el equipo Slim Tube para garantizar su operación segura y confiable y el GTN-I-029 asegura la integridad de los ítems de ensayo mediante su adecuada recepción, identificación, preparación, manipulación, almacenamiento y disposición final.

El procedimiento para la determinación de la mínima presión de miscibilidad es como sigue. Una vez se tiene el Slim Tube saturado con el crudo a evaluar, se inicia con un valor igual o mayor que la presión de burbuja. Se cuantifican los volúmenes producidos de crudo y gas y se estima el recobro. El siguiente valor de presión depende de si la eficiencia del desplazamiento fue superior o inferior al $95 \%$.

Se repite el procedimiento de preparación del equipo y se realiza el ensayo a una nueva condición. Al momento de graficar el porcentaje de recobro en función de la presión del ensayo se debe identificar de una manera clara una zona miscible y una inmiscible. La mínima presión de miscibilidad se determina por la intersección de las líneas de tendencia trazadas para cada una de estas zonas. 


\section{FLUIDOS A UTILIZAR}

Para la saturación del equipo Slim Tube se usó crudo del Campo Colombia en paz recombinado con gas de producción del mismo yacimiento. Durante las pruebas de miscibilidad se usó el gas hidrocarburo asociado a la producción. Personal técnico del Laboratorio PVT (Presión - Volumen - Temperatura) del Instituto Colombiano del petróleo realizó el muestreo de dichos fluidos en las facilidades de producción (Crude Production Facilities - CPF) del Campo.

\section{CONDICIONES DE OPERACIÓN}

Profesionales de ingeniería de yacimientos definieron las condiciones de operación en el equipo Slim Tube. La temperatura de los ensayos se fijó en $138^{\circ} \mathrm{F}$, la cual corresponde al valor promedio medido en las arenas basales del Campo Colombia en paz. El crudo que allí se produce tiene una gravedad API en el rango 28-30 grados, una relación gas aceite (Gas Oil Ratio - GOR) promedia de $440 \mathrm{scf} / \mathrm{RB}$ y una presión de saturación medida en laboratorio alrededor de 1,750 - 1,850 psi.

\section{DETERMINACIÓN DE LA MÍNIMA PRESIÓN DE MISCIBILIDAD}

El primer paso consiste en la adecuación y puesta a punto del equipo Slim Tube con base en el instructivo GTN-I-019. Como una actividad paralela e independiente se realiza la preparación y caracterización de los fluidos tanto crudo como gas. Se satura el medio poroso con crudo recombinado y se fijan las condiciones de operación tales como temperatura, presión y número de ensayos a realizar. Se usa una tasa de inyección de gas de $0.1 \mathrm{ml} / \mathrm{min}$ o menor para garantizar un diferencial de presión entre bomba y producción menor que 30 psi.

Para este proyecto particular se fijaron dos valores de presión por encima del punto de burbuja, 3,000 y 6,000 psi. Para cada uno de estos valores se desplazan 1.25 volúmenes porosos de gas y se colectan los efluentes en probetas conectadas a un medidor húmedo de gas Ritter TG 1. Se monitorean los volúmenes producidos de crudo y gas, la relación gas-aceite (Gas Oil Ratio GOR), la presión de bomba y se calcula el porcentaje de recobro.

\section{RESULTADOS Y DISCUSIÓN}

Mediante análisis Karl Fischer se verificó que el contenido de agua y sedimentos (Basic Sediments and Water - BSW) del crudo fuera menor que $1 \%$. Por esta razón no fue necesario deshidratarlo, ya que cumple con los requerimientos de laboratorio. Después se filtró por membrana con tamaño de poro de 0.5 micras previo a su inyección en el medio poroso del Slim Tube. Esto con el propósito de evitar su posible taponamiento con partículas sólidas en suspensión tales como arena, limadura, etc. Por último, mediante un análisis básico con la técnica del picnómetro se confirmó que la gravedad API del crudo estaba alrededor de 29 grados.

\section{CARACTERIZACIÓN DEL CRUDO RECOMBINADO Y DEL GAS DE PRODUCCIÓN}

Crudo recombinado: Para la determinación de Mínima Presión de Miscibilidad se recombinó crudo muerto con gas de producción, ambos tomados del Pozo Colombia en paz-001. Para ello se siguió el instructivo de ensayo UST-P-157-006 de Laboratorio PVT en el cual se usa como parámetro de control la relación gas aceite (Gas Oil Ratio - GOR) de campo. En el análisis de calidad realizado a la muestra ya recombinada se determinó su composición química y densidad, ver Tabla 1.

Tabla 1. Propiedades físicas del crudo

\begin{tabular}{lc}
\hline \multicolumn{2}{c}{ Crudo recombinado } \\
\hline GOR (Gas Oil Ratio) [scf/bl] & 440 \\
\hline Densidad [g/ml] & 0.88 \\
\hline API [grados] & 29 \\
\hline Peso Molecular C30+ [g/mol] & 580 \\
\hline Presión de burbuja [psi] & 1,850 \\
\hline Temperatura yacimiento [F] & 138 \\
\hline Factor volumétrico formación a 2,000 psi [RB/STB] & 1.25 \\
\hline
\end{tabular}

Gas de producción: A la muestra de gas de producción se le realizó análisis de cromatografía antes de su utilización en laboratorio. Como se puede observar en la Figura 1, el principal componente es el metano $\left(\mathrm{CH}_{4}\right)$ con un poco más del $84 \%$, luego etano con menos del $10 \%$ y en menor proporción propano con menos del 5 $\%$. Las impurezas como nitrógeno con menos del $1 \%$, lo cual no es significativo para incrementar la mínima presión de miscibilidad. La Gravedad Específica determinada en laboratorio fue 0.69 .

\section{RECOBRO DE CRUDO COLOMBIA EN PAZ-001 CON GAS DE PRODUCCIÓN}

Esta evaluación se realizó a dos valores de presión (3,000 y 6,000 psi), de acuerdo con la metodología de 
ensayo GTN-I-009. En la primera evaluación se obtuvo un recobro de $32.0 \%$, ver Figura 2, el cual es bajo y se debió a que la irrupción de gas ocurrió temprano, después de tan sólo 0.2 volúmenes porosos inyectados. A partir de este momento el GOR se incrementa, lo cual muestra que la presión mínima para que se presente miscibilidad entre estos dos fluidos es más alta.

En la segunda evaluación a una mayor presión, 6,000 psi, se obtuvo un recobro más alto, alrededor de $64.0 \%$. Pero al igual que en la anterior, de nuevo la irrupción de gas ocurrió temprano, después de 0.5 volúmenes porosos inyectados, ver Figura 3. A partir de este momento el GOR se incrementa lo cual muestra que la presión mínima para que se presente miscibilidad entre estos dos fluidos es más alta.

Ambas valores superan con creces la presión de diseño del sistema Gas Lift de 1,600 psi. Sin embargo, se eligió un primer valor de 3,000 psi debido a que el protocolo experimental recomienda iniciar por encima del punto de burbuja, la cual está en el rango 1750 1850 psi.

En la Figura 4 se puede observar el recobro de crudo a las dos presiones evaluadas. Como era de esperarse, se recupera más petróleo cuando se incrementa la presión, pero el valor de 6,000 psi aún se está por debajo de la presión mínima para que se presente miscibilidad, debido a que el recobro es inferior al 95 por ciento.

Sin embargo, en campo se esperaría un incremento en la productividad debido a mecanismos no miscibles. Uno de ellos sería la represurización en el área de drenaje de los pozos estimulados por la inyección cíclica (Huff and Puff). El otro sería una mejora de la movilidad por efecto de la solubilidad parcial del gas reinyectado en el petróleo.

Con base en los resultados obtenidos a partir de mediciones en equipo Slim Tube se estimó, mediante interpolación lineal, la eficiencia de desplazamiento a la presión máxima de operación del sistema Gas Lift. La cual fue del $18 \%$ pero no se debe interpretar como un valor escalable a campo debido a que los efectos gravitaciones y la heterogeneidad podrían hacer que este valor sea menor.

\section{ESTIMACIÓN DE LA MÍNIMA PRESIÓN DE MISCIBILIDAD}

En la Figura 5 se puede observar la Mínima Presión de Miscibilidad estimada para este gas. Se hace uso de los recobros medidos en laboratorio a las presiones de 3,000 y 6,000 psi para extrapolar el valor que se requeriría para lograr recuperar el $95 \%$ del petróleo. Allí se puede observar que en este caso se tendría que operar a más de 8,400 psi para estar en la región miscible o cerca de ella.

\section{CONCLUSIONES}

1. La eficiencia de desplazamiento de crudo con gas de producción a la presión máxima disponible en el sistema Gas Lift es baja debido a que está alrededor de un $18 \%$.

2. No sería viable la implementación de un proceso miscible en campo debido a que la Mínima Presión de Miscibilidad del gas de producción en este crudo se estimó en alrededor de 8,407 psi.

\section{REFERENCIAS}

1. Adel, I., Tovar, F. y Schechter, D. (2016). Fast-Slim Tube: A Reliable and Rapid Technique for the Laboratory Determination of MMP in $\mathrm{CO} 2$ Light Crude Oil Systems. SPE-179673-MS. SPE Improved Oil Recovery Conference held in Tulsa, Oklahoma, 11-13 April 2016. 12 p.

2. Al-Khazraji, A. y Awang, M. (2016). Evaluation of Miscible CO2 Flooding to Improve Oil Recovery in a Clastic Heterogeneous Reservoir. IADC/SPE-180529-MS. IADC/SPE Asia Pacific Drilling Technology Conference Held in Singapore, 22-24. 14 p.

3. Al Saadi, H., Mirza, M., Al Amri, B., Mucharam L., Nugroho W. y Al-Hinai K. (2016). Case Study in Middle East, Improve Heavy Oil Recovery and Production by Reservoir Modification as Alternative Technology to Steam and Miscible Gas Injection. SPE-179830-MS. SPE EOR Conference at Oil and Gas West Asia Held in Muscat, Oman, 21-23, March. 12 p.

4. BP Statistical Review of World Energy. (2016). bp.com/statisticalreview. \#BPstats. 48 p.

5. Cho, Y., Kim, T. y Lee, K. (2016). Modeling of CO2 EOR Process Combined with Intermediate Hydrocarbon Solvents for Higher Recovery Efficiency. SPE-179778-MS. SPE EOR Conference at Oil and Gas West Asia Held in Muscat, Oman, 21-23, March 2016. SPE Improved Oil Recovery Conference held in Tulsa, Oklahoma, 11-13 April. 12 p. 
6. Enhanced Oil Recovery (EOR) Dataset. (2016). Oil and Gas Journal online Research Center. PennEnergy Research Surveys, June. 14 p.

7. Ennin, E. y Grigg, R. (2016). CO2 Flooding and Minimum Miscibility Pressure Study in Texas Farnsworth Field. SPE-180854-MS. SPE Trinidad and Tobago Section Energy Resources Conference Held in Port of Spain, Trinidad and Tobago, 13-15 June. 13 p.

8. Rommerskirchen, R., Nijssen, P., Bilgili, H. y Sottmann, T. (2016). Additives for CO2 EOR Applications. SPE-181304-MS. SPE Annual Technical Conference and Exhibition Held in Dubai, UAE, 7-10 September. 13 p.

9. Rommerskirchen, R., Nijssen, P., Bilgili, H. y Sottmann, T. (2016). Reducing the Miscibility Pressure in Gas Injection Oil Recovery Processes. SPE-183389-MS. SPE Annual Technical Conference and Exhibition Held in Dubai, UAE, 7-10 September.14 p.

10. Zhang, K. (2016). Experimental y Numerical Investigation of Oil Recovery From Bakken Formation by Miscible $\mathrm{CO} 2$ Injection. SPE- 184446-STU. SPE Annual Technical Conference and Exhibition Held in Dubai, UAE, 29-28 September. 23 p.

11. Zhang, K., Seetahal, S., Alexander D., He, R., Lv, J., Wu, K. y Chen, Z. (2016). SPE-180857MS Correlation for CO2Minimum Miscibility Pressure in Tight Oil Reservoirs. SPE Trinidad and Tobago Section Energy Resources Conference Held in Port of Spain, Trinidad and Tobago, 13-15 June. 9 p.

\section{AGRADECIMIENTOS}

Los autores expresan su gratitud a Empresa Colombiana de Petróleos Ecopetrol S.A. y a la Universidad Industrial de Santander UIS por su constante apoyo durante el desarrollo de este trabajo técnico.

\section{CONVERSIONES}

Las equivalencias del Sistema Inglés de Unidades al Sistema Internacional de Unidades son las siguientes,

\begin{tabular}{cc}
\hline Inglés & Internacional \\
\hline $14.697 \mathrm{psi}$ & 14.5 bar \\
$\mathrm{T}[\mathrm{Fl}]$ & $1.8 * \mathrm{~T}[\mathrm{C}]+32$ \\
\hline
\end{tabular}

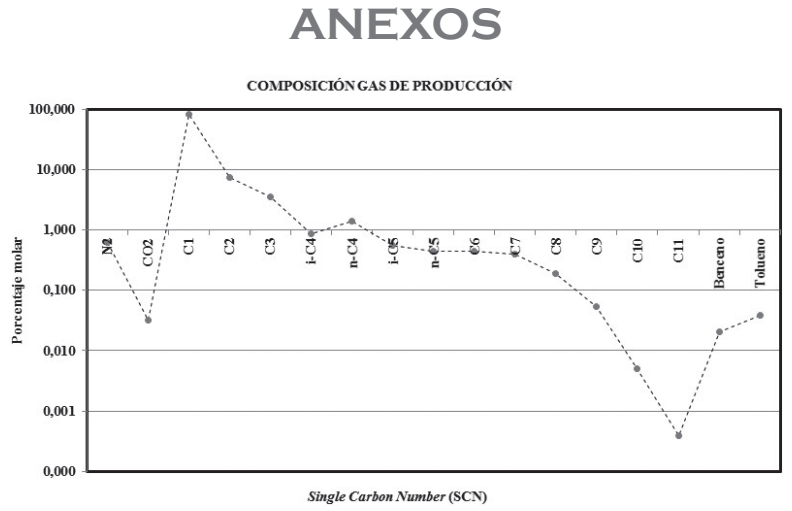

Figura 1. Composición gas de producción.

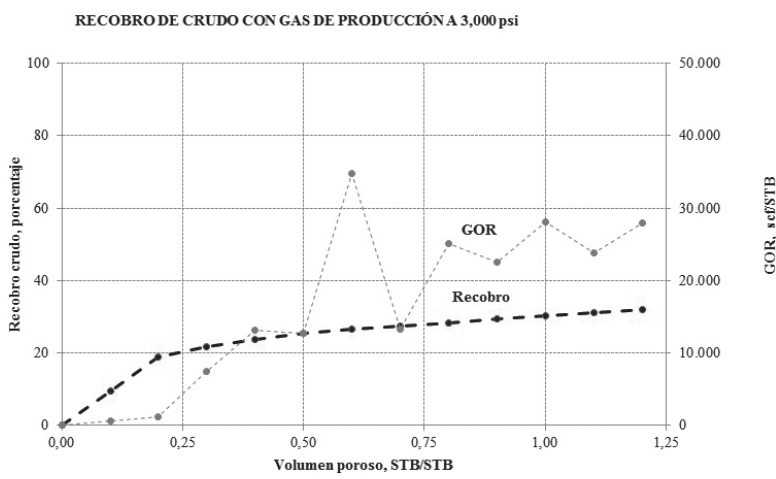

Figura 2. Recobro de crudo con gas de producción a 3,000 psi.

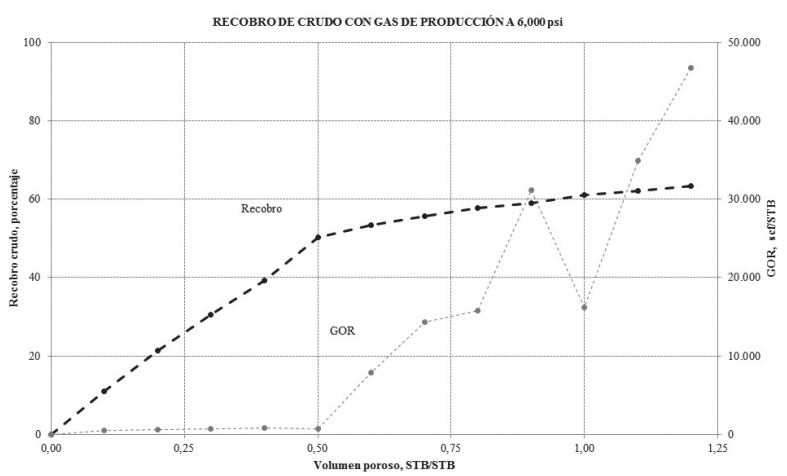

Figura 3. Recobro de crudo con gas de producción a 6,000 psi.

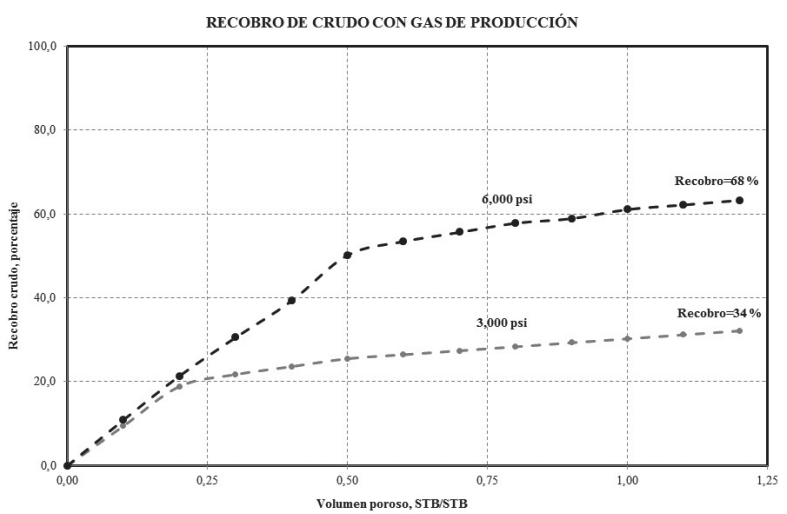

Figura 4. Recobro crudo con gas de produccón. 


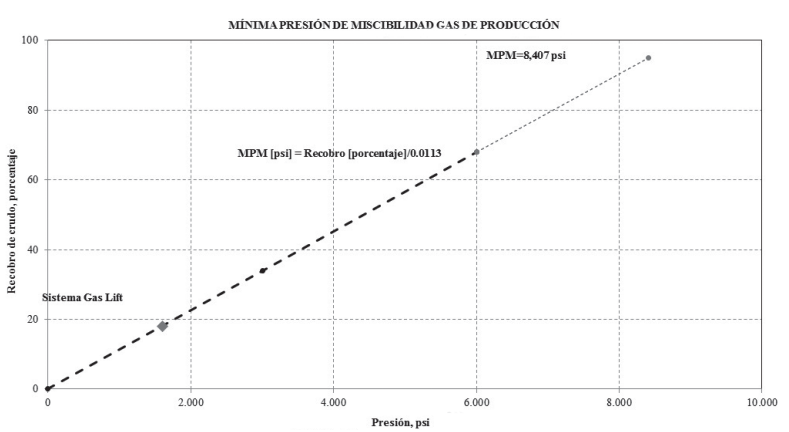

Figura 5. Estimación MPM gas de producción en crudo.

Recepción: 9 de Diciembre de 2016

Aceptación: 27 de Abril de 2017 